SLAC-PUB-8792

March 2001

\title{
Recent Tests of QCD at SLD*
}

\author{
David Muller \\ Representing The SLD Collaboration** \\ Stanford Linear Accelerator Center \\ Stanford University, Stanford, CA 94309
}

\begin{abstract}
We present selected results on strong interaction physics from the SLD experiment at the SLAC Linear Collider. We report on several new studies of 3- and 4-jet hadronic $Z^{0}$ decays, in which jets are identified as quark, antiquark or gluon. The gluon energy spectrum is measured over the full kinematic range, providing an improved test of QCD and limits on anomalous $b b g$ and $b b g$ couplings. The parity violation in $Z^{0} \rightarrow b \bar{b} g$ decays is consistent with electroweak theory plus QCD. New tests of T- and CP-conservation at the $b b g$ vertex are performed. An improved measurement of the rate of gluon splitting into $b \bar{b}$ pairs yields $g_{b \bar{b}}=0.00244 \pm 0.00059$ (stat.) \pm 0.00034 (syst.). We also present a number of new results on jet fragmentation into identified hadrons. The $B$ hadron energy spectrum is measured over the full kinematic range using a new, inclusive technique, allowing stringent tests of predictions for its shape and a precise measurement of $\left\langle x_{B}\right\rangle=0.710 \pm 0.003$ (stat.) \pm 0.006(syst.) (Preliminary). A detailed study of correlations in rapidity $y$ between pairs of identified $\pi^{ \pm}, K^{ \pm}$and $\mathrm{p} / \overline{\mathrm{p}}$ confirms that strangeness and baryon number are conserved locally, and shows local charge conservation between meson-baryon and strange-nonstrange pairs. Flavor-dependent long-range correlations are observed for all combinations of these hadron species, yielding new information on leading particle production. The first study of correlations using rapidities signed such that $y>0$ corresponds to the quark direction provides additional new insights into fragmentation, including the first direct observation of baryon number ordering along the $q \bar{q}$ axis.
\end{abstract}

\section{Presented at Frontiers in Comtemporary Physics II, 5-10 March, 2001, Nashville, Tennessee.}

*This work was supported in part by DOE grant DE-AC03-76SF00515. 


\section{Introduction}

We present an overview of a number of recent measurements from the SLD experiment, using hadronic decays of $Z^{0}$ bosons produced in $e^{+} e^{-}$annihilations. First we present a number of precision tests of QCD in the perturbative regime using 3- and 4-jet final states. The goal here is sensitivity to radiative corrections to the reaction $e^{+} e^{-} \rightarrow Z^{0} / \gamma \rightarrow q \bar{q}(g)$ induced by known effects, such as the large mass of the $b$-quark, or by new physics. Such effects are expected at the few percent level, so the effects of higher order gluon radiation must be understood or suppressed at this level. Since we do not observe partons directly, rather the jets of particles into which they fragment, it is essential to understand the properties of these jets. Here we present two new, detailed studies of jet formation involving identified hadrons measured over a wide energy range.

A key to improving upon existing studies of multijet events has been the identification of the parton species that initiated a particular jet, or the identification of the primary $q$ flavor in $e^{+} e^{-} \rightarrow q \bar{q}$. In practice, this is done by identifying jets containing $b$ or $\bar{b}$ quarks, giving the added benefits of important input into measurements such as electroweak parameters $\left(R_{b}\right.$ and $\left.A_{b}\right)$ in $Z^{0}$ decays and bottom production in hadron-hadron collisions, and sensitivity to new physics that couples more strongly to heavier quarks.

Experimental studies of the structure of 3-jet events have typically used energy and angle distributions of energy-ordered jets. Since the gluon is expected to be the lowestenergy jet in most events, this suffices to confirm the $q \bar{q} g$ origin of such events and to determine the gluon spin. The identification of the three jets in such events would allow more complete and stringent tests of QCD. Here we present a study of 3-jet final states in which two of the jets are tagged as $b$ or $\bar{b}$ jets. The remaining jet is tagged as the gluon jet and its energy spectrum studied over the full kinematic range [1]. Adding a tag of the charge of the $b$ or $\bar{b}$ jet, and exploiting the high electron beam polarization of the SLC, we measure [2] two angular asymmetries sensitive to parity violation in the $Z^{0}$ decay, and also construct new tests of $\mathrm{T}$ - and CP-conservation at the $b b g$ vertex.

The rate of secondary heavy flavor production via gluon splitting, $g \rightarrow c \bar{c}, g \rightarrow b \bar{b}$ is a sensitive test of QCD, as it is suppressed strongly by the mass of the heavy quark, but is still expected to be the dominant source of secondary heavy hadrons. Here we present a measurement of the $g \rightarrow b \bar{b}$ rate [3] that is complementary to and more precise than other measurements.

The process of jet formation is not understood quantitatively, due to the difficulty of perturbative calculations in this soft regime. A number of phenomenological models have been developed, and it is essential to understand the properties of jets empirically in order to test these models and encourage theoretical development. Since jets are used in many precision tests of electroweak and strong physics (e.g. those described here), and will constitute both the largest signal for and the background to any heavy particles discovered in the future, our understanding, even if only through models, must be as complete as possible.

The production of heavy hadrons from heavy primary quarks is relatively easy to calculate perturbatively due to the cutoff introduced by the large quark mass. Several calculations and model predictions for the energy spectrum of bottom hadrons now exist and await 
precise testing. Experimental studies of the $B$-hadron spectrum have been limited by the efficiency for reconstructing the energies $E_{B}$ of individual $B$ hadrons with good resolution, especially for low-energy $B$ hadrons. Here we present a study [4] of the $E_{B}$ distribution using a novel kinematic technique and only charged tracks. The high efficiency and good resolution for all $E_{B}$, results in a measurement covering the full kinematic range.

Lighter identified particles are also an active field of study. The production of strange particles and baryons is of particular interest as they must be produced in strange-antistrange or baryon-antibaryon pairs, and the mechanism of their pair production can yield insights into the fragmentation process. Previous studies of rapidity correlations have shown that the conservation of such quantum numbers is predominantly local or short-range, i.e. the two particles are produced close together in the jet phase space. There is also evidence for long-range correlations from the leading particles in the two hemispheres of an event that contain the primary quark and antiquark. Here we present detailed studies [5] of shortand long-range correlations between identified $\pi^{ \pm}, K^{ \pm}$and $\mathrm{p} / \overline{\mathrm{p}}$ that improve substantially upon previous measurements. In addition, we use the SLC beam polarization to tag the quark hemisphere in each event and study for the first time rapidities signed such that positive (negative) rapidity corresponds to the (anti)quark hemisphere. Ordered differences in signed rapidity provide further unique insights into jet fragmentation, in particular being sensitive to local ordering of quantum numbers along the $q \bar{q}$ axis.

Unless otherwise noted, these measurements use the entire SLD hadronic sample of 550,000 events recorded from 1993 to 1998. Each exploits some of the unique feaures of the SLC/SLD program, such as the highly longitudinally polarized electron beam, small transverse beam size, and excellent vertex detection and particle identification. The magnitude of the beam polarization averaged $73 \%$, providing high sensitivity to parity violating observables and a quark hemisphere tag of $73 \%$ purity. The collision region typically measured $0.8 \mu \mathrm{m}$ vertically by $1.8 \mu \mathrm{m}$ horizontally, which combined with the vertex detector gives a transverse impact parameter resolution of $\sigma_{\delta}=8 \oplus 29 / p \sin ^{3 / 2} \theta \mu \mathrm{m}$, providing excellent heavy- and light-flavor tagging. We use a topological algorithm [6] that finds secondary vertices cleanly and efficiently, and also measures the flight direction independent of the momenta of the tracks in the vertex. Cutting on the vertex mass provides $B$ (charmed) hadron samples of up to $98 \%(70 \%)$ purity. In addition we consider the number of tracks in an event or jet, $n_{\text {sig }}^{(m)}$, that have a normalized transverse impact parameter wrt the in-

teraction point (IP) of $\delta / \sigma_{\delta}>m$. Heavy hadron decays tend to give $n_{\text {sig }}^{(m)}>0$, and the absence of such tracks provides an efficient light-flavor tag.

\section{Three-Jet Event Structure}

Hadronic events [1] with exactly 3 jets (JADE algorithm, $y_{\text {cut }}=0.02$ ) are selected. Jet energies $E_{i}$ are calculated from the interjet angles [7], and the jets are energy ordered: $E_{1}>E_{2}>E_{3}$. We require a topological vertex in exactly two of the three jets, with at least one having a mass greater than $2 \mathrm{GeV} / \mathrm{c}^{2}$, and the remaining jet is tagged as the gluon jet. This yields 6504 events in our 1997-8 sample with an estimated purity of correctly tagged gluon jets of $94 \%$. In $3.3 \%(14.4 \%)$ of these events, jet $1(2)$, the (second) highest energy 


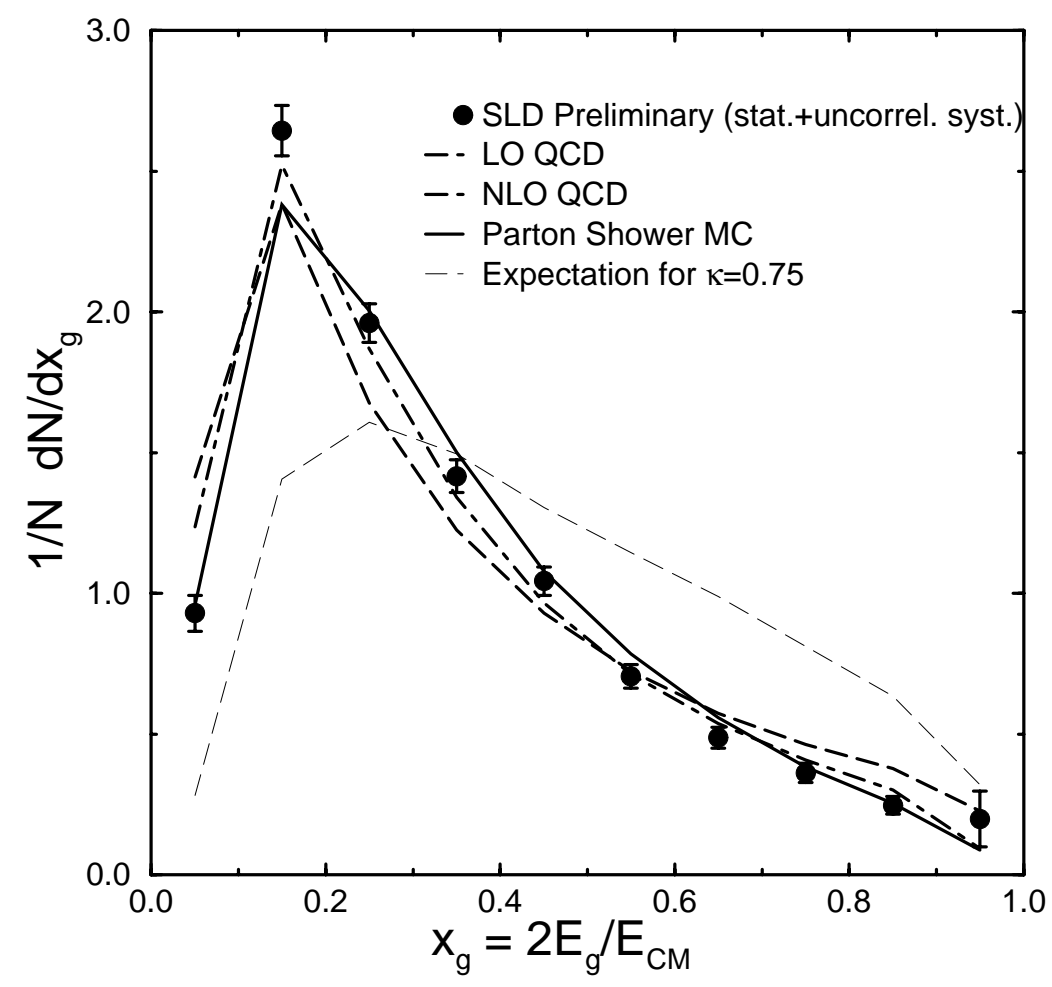

Figure 1: The fully corrected scaled gluon energy distribution (dots). The predictions of leading- and next-to-leading-order QCD and of a parton shower simulation are shown as the dashed, dot-dashed and solid lines, respectively. The thin dashed line shows the prediction for an anomalous chromomagnetic coupling at the $b b g$ vertex with relative strength 0.75 .

jet, is tagged as the gluon jet, giving coverage over the full kinematic range.

The distribution of the scaled gluon energy $x_{g}=2 E_{g} / \sqrt{s}$ is corrected for non- $b \bar{b} g$ and mistag backgrounds, selection efficiency and resolution. The fully corrected spectrum, fig. 1, shows the expected falling behaviour with increasing $x_{g}$. It is cut off at low $x_{g}$ by the finite $y_{\text {cut }}$ value. Also shown are the predictions of first and second order QCD [8]; both describe the data in general, but not in detail. The prediction of the JETSET [9] parton shower comes quite close to reproducing the data. We thus confirm the prediction of QCD, although higher order effects are clearly important in the intermediate gluon energy range, $0.2<x_{g}<0.4$.

The $x_{g}$ spectrum is particularly sensitive to the presence of an anomalous chromomagnetic term in the strong interaction Lagrangian (see fig. 1). A fit of the theoretical prediction [10] including an anomalous term parametrized by a relative coupling $\kappa$, yields a value of $\kappa=-0.02 \pm 0.05$ (Preliminary), consistent with zero, and corresponding to $95 \%$ C.L. limits on such contributions to the $b b g$ coupling of $-0.11<\kappa<0.07$.

Parity violation in 3-Jet events was studied using two angles, the polar angle of the quark with respect to the electron beam direction $\theta_{q}$, and the angle between the quark-gluon and quark-electron beam planes $\chi=\cos ^{-1}\left(\hat{p}_{q} \times \hat{p}_{g}\right) \cdot\left(\hat{p}_{q} \times \hat{p}_{e}\right)$. The cosine $x$ of each of these angles should be distributed as $1+x^{2}+2 A_{P} A_{Z} x$, where the $Z^{0}$ polarization $A_{Z}=\left(P_{e}-A_{e}\right) /\left(1-P_{e} A_{e}\right)$ depends on that of the $e^{-}$beam $P_{e}$, and $A_{e}$ and $A_{P}=A_{0} A_{q}$ are predicted by QCD plus electroweak theory. 

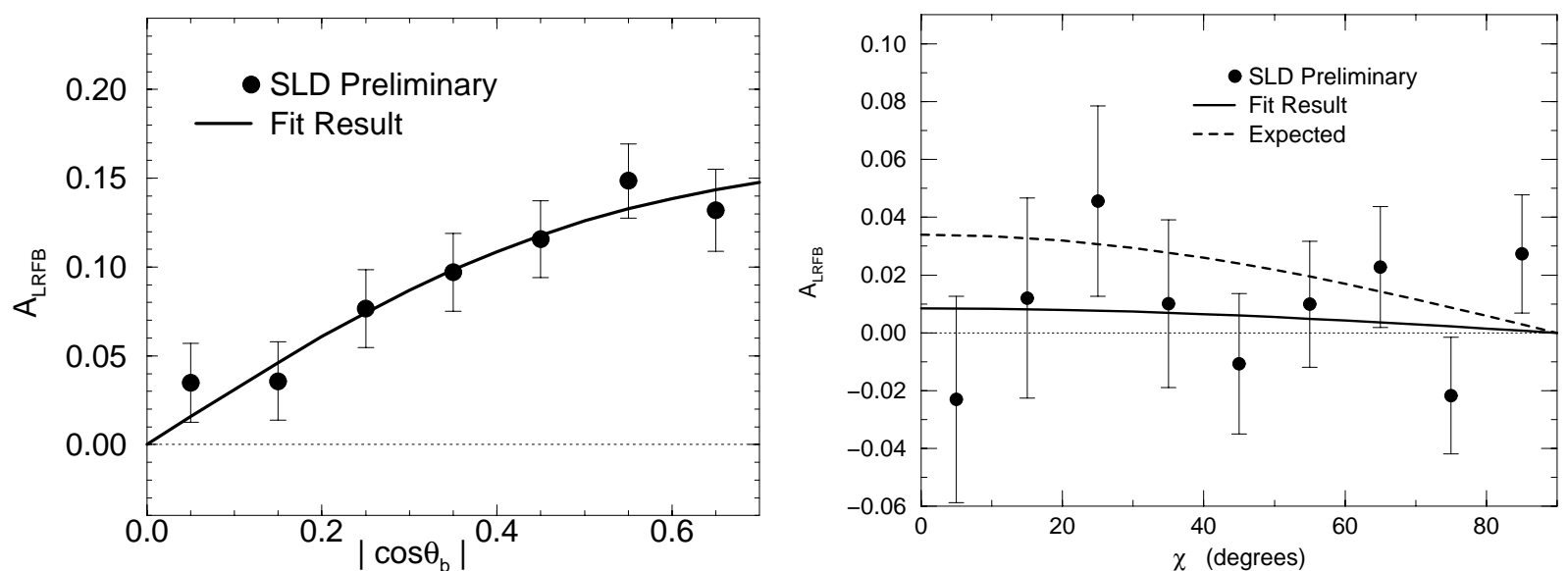

Figure 2: Left-right-forward-backward asymmetry of (left) the $b$-quark polar angle and (right) the angle $\chi$ in 3 -jet $Z^{0} \rightarrow b \bar{b} g$ decays. The solid lines are the results of fits; the dashed line is the QCD prediction.

Three-jet events (Durham algorithm, $y_{c u t}=0.005$ ) are selected and energy ordered. The 14,658 events containing a secondary vertex with mass above $1.5 \mathrm{GeV} / \mathrm{c}^{2}$ in any jet are kept, having an estimated $b \bar{b} g$ purity of $85 \%$. We calculate the momentum-weighted charge of each jet $j, Q_{j}=\Sigma_{i} q_{i}\left|\vec{p}_{i} \cdot \hat{p}_{j}\right|^{0.5}$, using the charge $q_{i}$ and momentum $\vec{p}_{i}$ of each track $i$ in the jet. We assume that the highest-energy jet is not the gluon, and tag it as the $b(\bar{b})$ if $Q=Q_{1}-Q_{2}-Q_{3}$ is negative (positive). We define the $b$-quark polar angle by $\cos \theta_{b}=-\operatorname{sign}(Q)\left(\hat{p}_{e} \cdot \hat{p}_{1}\right)$.

The left-right-forward-backward asymmetry $A_{L R F B}^{b}$ in $\cos \theta_{b}[2]$ is shown as a function of $\left|\cos \theta_{b}\right|$ in fig. 2. The clear asymmetry increases with increasing $\left|\cos \theta_{b}\right|$ in the expected way. A fit to the data yields an asymmetry parameter of $A_{P}=0.914 \pm 0.053$ (stat.) \pm 0.063 (syst.), consistent with the QCD prediction of $A_{P}=0.93 A_{b}=0.87$.

We then tag one of the two lower energy jets as the gluon jet: if jet 2 has $n_{\text {sig }}^{(3)}=0$ and jet 3 has $n_{\text {sig }}^{(3)}>0$, then jet 2 is tagged as the gluon; otherwise jet 3 is tagged as the gluon. We construct the angle $\chi$, and $A_{L R F B}^{\chi}$ is shown as a function of $\chi$ in fig. 2. Here we expect only a small deviation from zero as indicated by the dashed line on fig. 2. Our measurement is consistent with the prediction, as well as with zero. A fit yields $A_{\chi}=-0.014 \pm 0.035 \pm 0.002$, to be compared with an expectation of -0.064 .

Using these fully tagged events, we can construct observables that are formally odd under time and/or CP reversal. For example, the triple product $\cos \omega^{+}=\vec{\sigma}_{Z} \cdot\left(\hat{p}_{1} \times \hat{p}_{2}\right)$, formed from the directions of the $Z^{0}$ polarization $\vec{\sigma}_{Z}$ and the highest- and second highest-energy jets, is $T_{N}$-odd and CP-even. Since the true time reversed experiment is not performed, this quantity could have a nonzero $A_{L R F B}$, and we have previously set a limit [7] using events of all flavors. A calculation [11] including Standard Model final state interactions predicts that $A_{L R F B}^{\omega^{+}}$is largest for $b \bar{b} g$ events, but is only $\sim 10^{-5}$. The fully flavor-ordered triple product $\cos \omega^{-}=\vec{\sigma}_{Z} \cdot\left(\hat{p}_{q} \times \hat{p}_{\bar{q}}\right)$ is both $T_{N^{-o d d}}$ and CP-odd.

Our measured $A_{L R F B}^{\omega^{+}}$and $A_{L R F B}^{\omega-}$ are shown in fig. 3. They are consistent with zero at all $|\cos \omega|$. Fits to the data yield $95 \%$ C.L. limits on any $T_{N}$-violating and CP-conserving or CP-violating asymmetries of $-0.045<A_{T}^{+}<0.016$ or $-0.082<A_{T}^{-}<0.012$, respectively. 


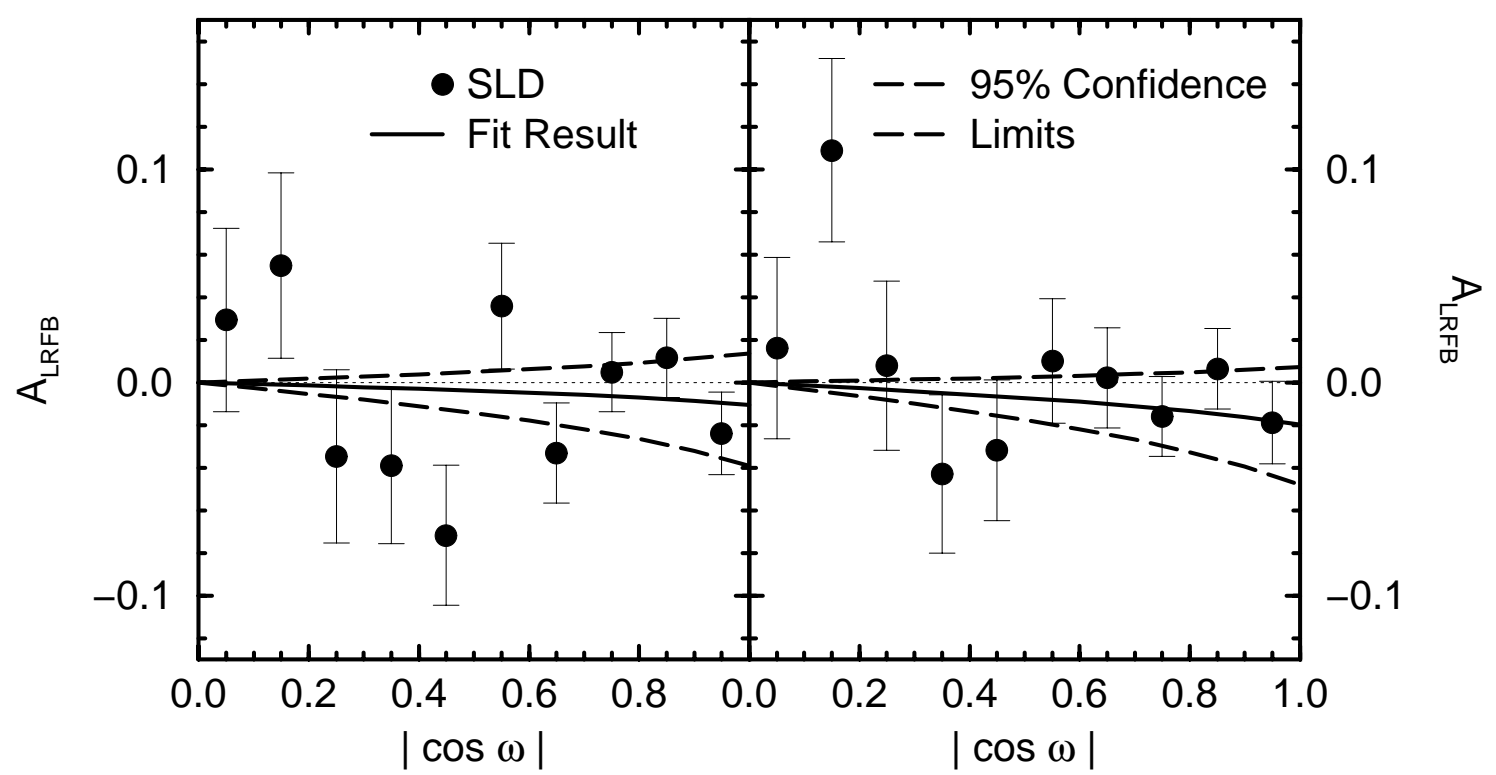

Figure 3: Left-right-forward-backward asymmetries of the energy- (left) and flavor-ordered (right) triple product. The (dashed) lines represent fits to the data (95\% confidence limits).

\section{Gluon Splitting into a $b \bar{b}$ Pair}

Candidate events containing a gluon splitting into a $b \bar{b}$ pair, $Z^{0} \rightarrow q \bar{q} g \rightarrow q \bar{q} b \bar{b}$, where the initial $q \bar{q}$ can be any flavor, are required to have 4 jets according to the Durham algorithm with $y_{\text {cut }}=0.008$. A secondary vertex is required in each of the two jets with the smallest opening angle in the event, yielding 1514 events. This sample is dominated by background, primarily from $Z^{0} \rightarrow b \bar{b} g(g)$ events and events with a gluon splitting into a $c \bar{c}$ pair.

A large component of the former background is $Z^{0} \rightarrow b \bar{b} g$ events in which the $b$ or $\bar{b}$ jet is split into two jets by the jetfinder, and two distinct vertices from the same $B$-hadron decay are found. Since the small beam spot allows the vertex flight directions to be measured precisely, the angle between the two flight directions from this background source tends to be small. Additional discriminating variables, including the sum of the energies of the two jets, the angle between the plane formed by the two selected jets and that formed by the other two jets in the event, and the vertex masses and momenta are used as inputs to a neural network [3]. The distribution of the network output $O$ is shown in fig. 4 . The simulation and data are consistent, and the simulated baackground falls rapidly with increasing $O$. A cut of $O>0.7$ keeps 79 events, with an estimated background of $41.9 \pm 1.8$ events. Using this and the estimated efficiency for selecting $g \rightarrow b \bar{b}$ splittings of $5.0 \%$ yields a measured fraction of hadronic events containing such a splitting of

$$
g_{b \bar{b}}=0.00244 \pm 0.00059 \text { (stat.) } \pm 0.00034 \text { (syst.). }
$$

The systematic error is dominated by the modelling of the gluon splitting process, which is estimated using a number of models. Due to the excellent efficiency for finding vertices from low-energy $B$ hadrons, we are less sensitive to this than other experiments. 


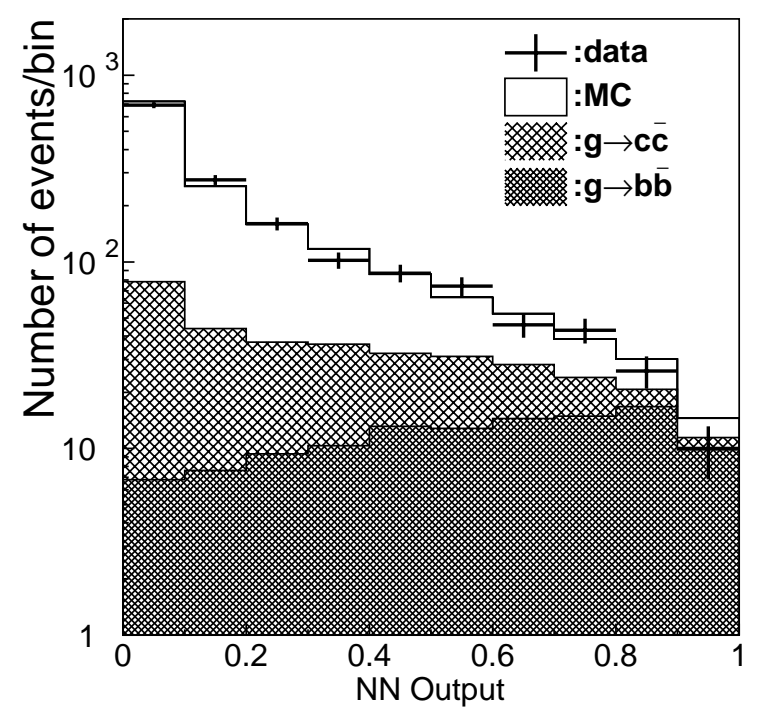

Figure 4: Distribution of the neural network output for candidate gluon splitting events (crosses). The signal and background shapes expected from the simulation are indicated.

\section{The $B$ Hadron Energy Spectrum}

Any secondary vertex with $m>2 \mathrm{GeV} / \mathrm{c}^{2}$ is considered as a candidate $B$-hadron vertex, with flight direction along the line joining the IP and the vertex position. The momentum $\vec{P}_{c h g}$ and mass $M_{c h g}$ of the set of tracks in the vertex (assigned the charged pion mass) is calculated, and $P_{\text {chgt }}$, transverse to the flight direction, is equated with $P_{0 t}$ of the "missing" momentum. Two more quantities, $M_{0}$ and $P_{0 l}$, are needed to determine the $B$ hadron energy $E_{B}$. Assuming a $B$ hadron mass of $M_{B}$ eliminates one of these unknowns, and also allows an upper limit to be calculated on the missing mass $M_{0}$ :

$$
M_{0 \max }^{2}=M_{B}^{2}-2 M_{B} \sqrt{M_{c h g}^{2}+P_{t}^{2}}+M_{c h g}^{2} .
$$

Using $M_{B}=5.28 \mathrm{GeV} / \mathrm{c}^{2}$, equating $M_{0}^{2}$ with $M_{0 \max }^{2}$ and solving for $E_{B}$ provides a good estimate of the true $E_{B}$. The simulated energy resolution is best for vertices with small $M_{0 \max }^{2}$; it approaches $6 \%$ as $M_{0 \max }^{2} \rightarrow 0$ and does not degrade rapidly with increasing $M_{0 \max }^{2}$ due to the strong tendency for the true $M_{0}$ to cluster near the maximum value.

A cut is placed on $M_{0 \max }^{2}$ that depends on the measured $E_{B}$ such that the simulated efficiency for selecting $B$ vertices is independent of energy; it averages $4 \%$ and is $>3.5 \%$ for $E_{B}>8 \mathrm{GeV}$. A sample of 4164 vertices is selected with an estimated purity of $99.0 \%$. The simulated energy resolution is $10 \%$ on average, roughly independent of $E_{B}$. The raw distribution of the scaled energy $x_{B}=E_{B} / E_{\text {beam }}$ is shown in fig. 5 , and covers the entire kinematic range from the $B$-hadron mass $\left(x_{B} \approx 0.12\right)$ to the beam energy. Our simulation uses the JETSET program with the Peterson fragmentation option and $\epsilon_{b}=0.006$, and predicts a peak position consistent with that of the data, but a significantly larger width.

The correction of these data to obtain the true $x_{B}$ distribution depends on the form assumed for the true distribution, due to the rapid variation of the distribution on the scale of the bin size. We therefore test several hypothesized shapes by weighting our 


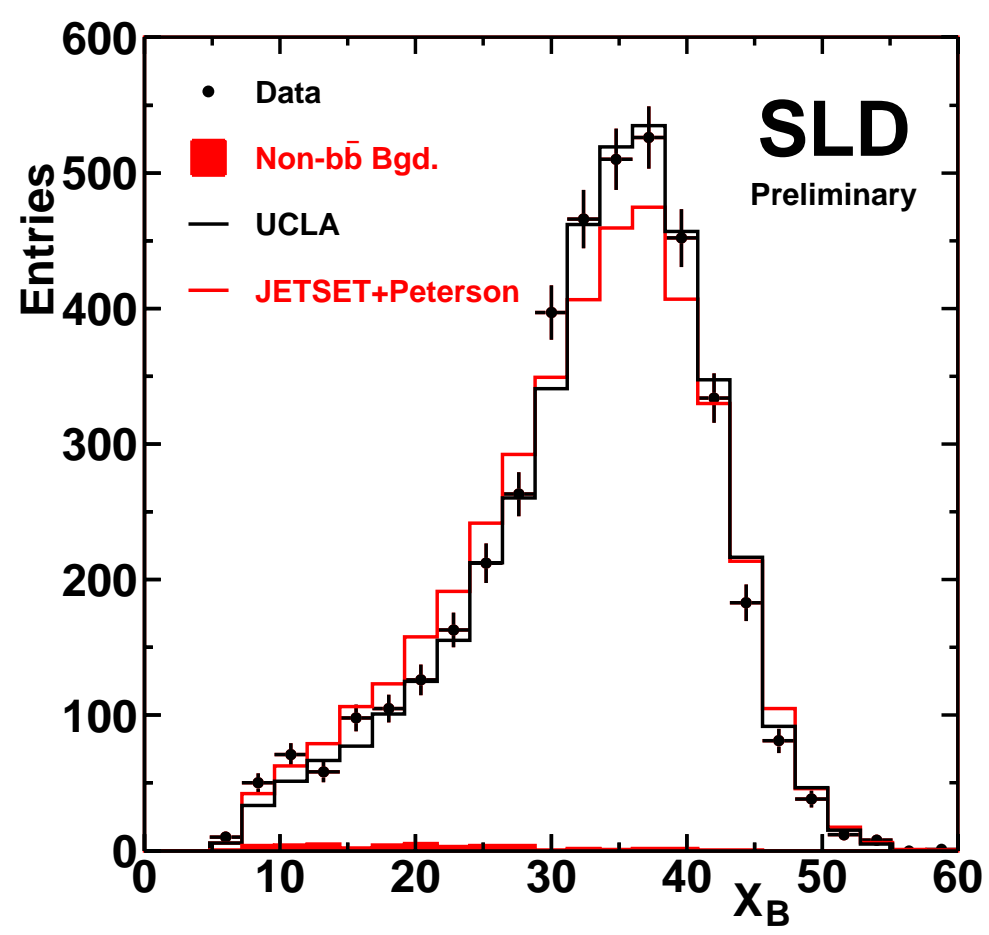

Figure 5: Uncorrected distribution of reconstructed $B$-hadron energies (dots). The gray (black) histogram is the prediction of the JETSET+Peterson (UCLA) simulation.

simulated events to reproduce a given function at the generator level, and comparing the corresponding detector level prediction with the data in fig. 5 using a binned $\chi^{2}$.

We first consider $B$ fragmentation models within the JETSET simulation. For the Peterson function we fit $\epsilon_{b}=0.006$; the $\chi^{2}$ of 62 for 16 degrees of freedom is unacceptably high. We also exclude [4] the models of Braaten et al., and Collins and Spiller; those of the Lund group, Bowler, and Kartvelishvili are able to describe the data. The UCLA and HERWIG models have no explicit free parameters governing $B$ hadron production; the UCLA model (see fig. 5) is consistent with the data; the HERWIG model is not. We also test several ad hoc functional forms $f\left(x_{B}\right)$ of the observable $x_{B}$ itself. Most of our test functions [4] are not able to describe the data. Four, the Peterson function, two generalizations thereof, and a sixth order polynomial, are found that do describe the data.

We subtract background and obtain the true distribution $D_{i}^{\text {corr }}=\Sigma_{k} M_{i k} D_{k}^{\text {meas }} / \epsilon_{i}$, using an efficiency $\vec{\epsilon}$ and unfolding matrix $\mathbf{M}$ simulated assuming in turn each of the four models and four functional forms that are consistent with the data. The model dependence of the procedure is thus explicit, and in fig. 6 we show in each bin $i$ the average of the eight $D_{i}^{\text {corr }}$, and the error bar includes their rms deviation, which is substantial at high $x_{B}$. The corrected distribution is, by construction, smoother than the measured distribution, and the errors provide a $1 \sigma$ envelope within which any acceptable prediction must fall.

From these eight forms we extract a measurement of the mean value of the scaled energy,

$$
\left\langle x_{B}\right\rangle=0.710 \pm 0.003 \text { (stat.) } \pm 0.005 \text { (syst.) } \pm 0.004(\text { rms })
$$

(Preliminary). This is the most precise measurement that takes the shape dependence into account; this uncertainty is small since we are able to exclude a wide range of shapes. 


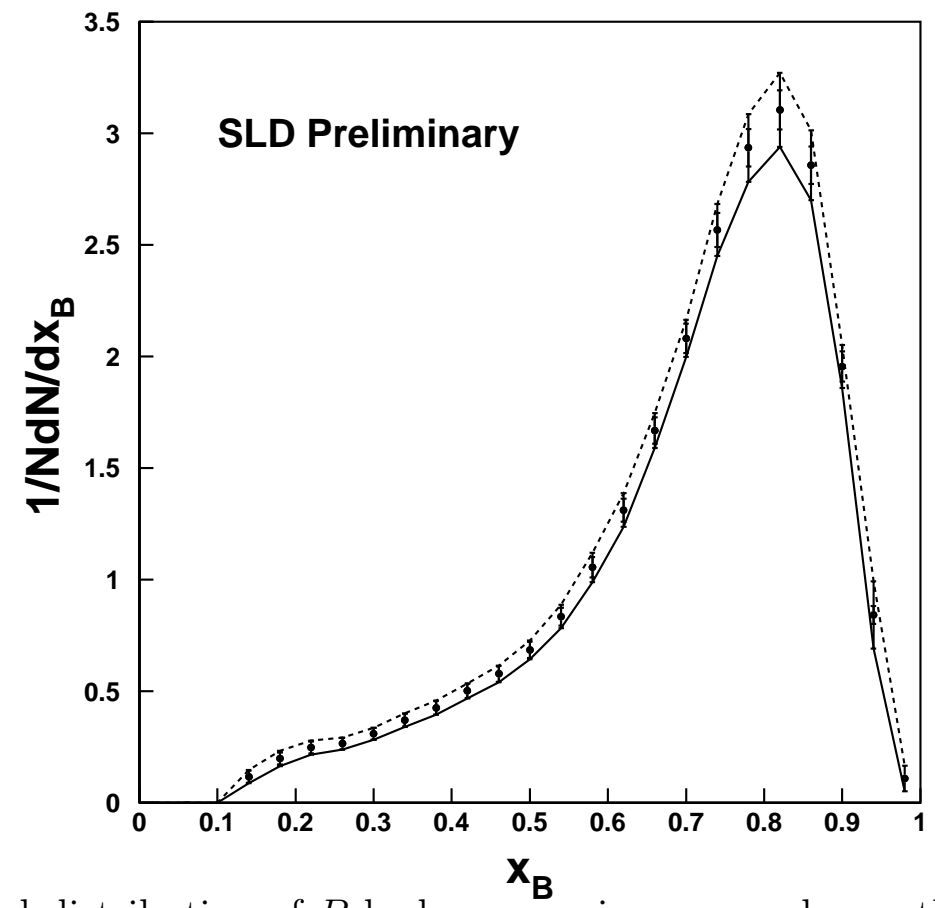

Figure 6: Corrected distribution of $B$-hadron energies averaged over the eight acceptable shapes. The outer error bars include the rms deviation among these shapes.

\section{Rapidity Correlations}

Here [5] we use all hadronic events, and subsamples tagged using $n_{\text {sig }}=0,1-3,>3$ as light-(uds), $c^{-}$, and $b$-flavor, respectively (purities 88\%, 39\%, and 93\%). For charged tracks identified as $\pi^{ \pm}, K^{ \pm}$or $\mathrm{p} / \overline{\mathrm{p}}$, the rapidity $y=0.5 \ln \left(\left(E+p_{\|}\right) /\left(E-p_{\|}\right)\right)$is calculated using the measured energy and momentum along the thrust axis $p_{\|}$. For each pair of tracks in an event the absolute rapidity difference $|\Delta y|=\left|y_{1}-y_{2}\right|$ is considered. Figure 7 shows $|\Delta y|$ distributions for (top right) identified $K^{+} K^{-}$pairs and $K^{+} K^{+} / K^{-} K^{-}$pairs. Assuming the latter to be uncorrelated, the excess of the former at low $|\Delta y|$ (short-range correlation) indicates that strangeness conservation is local in the jet fragmentation process. This is also the case for baryon number and electric charge, as is well known.

Also visible in fig. 7 are short-range correlations for all three unlike pair combinations. The excellent particle identification excludes the possibility that these are due to $\pi \pi$ pairs with one misidentified pion. This first direct observation of a fundamental feature of jet fragmentation, that charge can be conserved locally between a meson and a baryon, or between a strange and nonstrange particle, suggests charge ordering along the entire fragmentation chain. The high statistics and wide momentum coverage allow detailed measurements [5]: the JETSET model is found to describe the amplitude and range of the observed correlations except for $K \mathrm{p}$ pairs; this is true in six bins of momentum, and we conclude that, within the context of the JETSET model, the correlations are scale invariant.

We study long-range correlations, expected from leading particle production, using tracks with $p>9 \mathrm{GeV} / \mathrm{c}$. Differences between the opposite- and same-charge $|\Delta y|$ distributions for pairs of such tracks are shown for the three flavor-tagged samples in fig. 8. A 


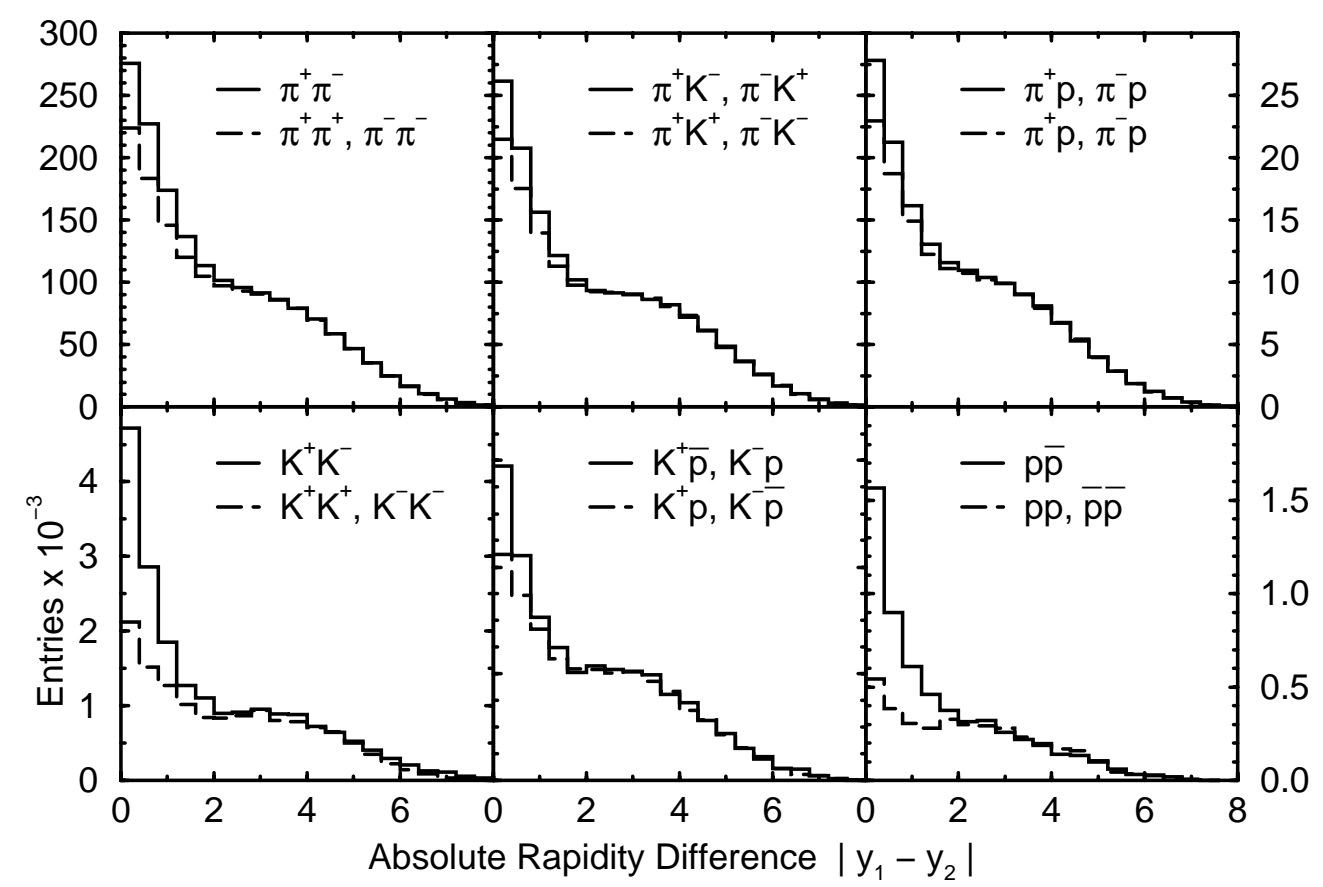

Figure 7: Distributions of absolute rapidity difference for opposite-charge (histogram) and same-charge (dashed histrogram) pairs of identified charged tracks in hadronic $Z^{0}$ decays.

large $K^{+} K^{-}$correlation is seen in light-flavor events, as expected from $s \bar{s}$ events in which the $s(\bar{s})$ jet produces a leading $K^{-}\left(K^{+}\right)$. Everywhere else, we expect dilutions from short-range correlations - e.g. each leading baryon will be accompanied by a subleading antibaryon with similar $y$. However significant correlations are observed for all pair combinations in light flavor events, providing new information on leading particle production. The JETSET model predictions are consistent [5], except for the $\pi K$ correlation. Flavor tagging is essential for these measurements; decays of $D$ hadrons contribute to most correlations; in particular they give a $\pi K$ anticorrelation that would mask the signal from light flavors.

We give $y$ a meaningful sign using the beam polarization to tag the quark hemisphere in each event (purity 73\%). The thrust axis is then signed so that $y>0(y<0)$ in the (anti)quark-tagged hemisphere. Ordered rapidity differences can then be defined; for oppositely charged pairs we define $\Delta y^{+-}=y_{+}-y_{-}$. A $\Delta y^{+-}$distribution can be studied simply by comparing its positive and negative sides. In fig. 9 we show the distributions of $\Delta y^{+-}$, along with the differences between the two sides, for $\pi^{+} \pi^{-}, K^{+} K^{-}$and $\mathrm{p} \overline{\mathrm{p}}$ pairs. At long-range $\left(\left|\Delta y^{+-}\right|>3\right)$, a large negative difference is observed for $K^{+} K^{-}$pairs, as expected from leading $K^{ \pm}$; a corresponding small positive difference is visible for $\mathrm{p} \overline{\mathrm{p}}$ pairs, but a small negative difference for $\pi^{+} \pi^{-}$pairs is not. Flavor tagging again proves beneficial: positive differences are present only in the $c$-tagged sample, and are explained by the leading charmed hadrons; a small negative difference is observed in the light-flavor sample [5].

The large positive difference observed for p $\bar{p}$ pairs at low $\left|\Delta y^{+-}\right|$is the first direct observation of another fundamental feature of jet fragmentation, the ordering of baryon number along the $q \bar{q}$ axis. That is, the proton in a correlated p $\bar{p}$ pair 'knows' and prefers 


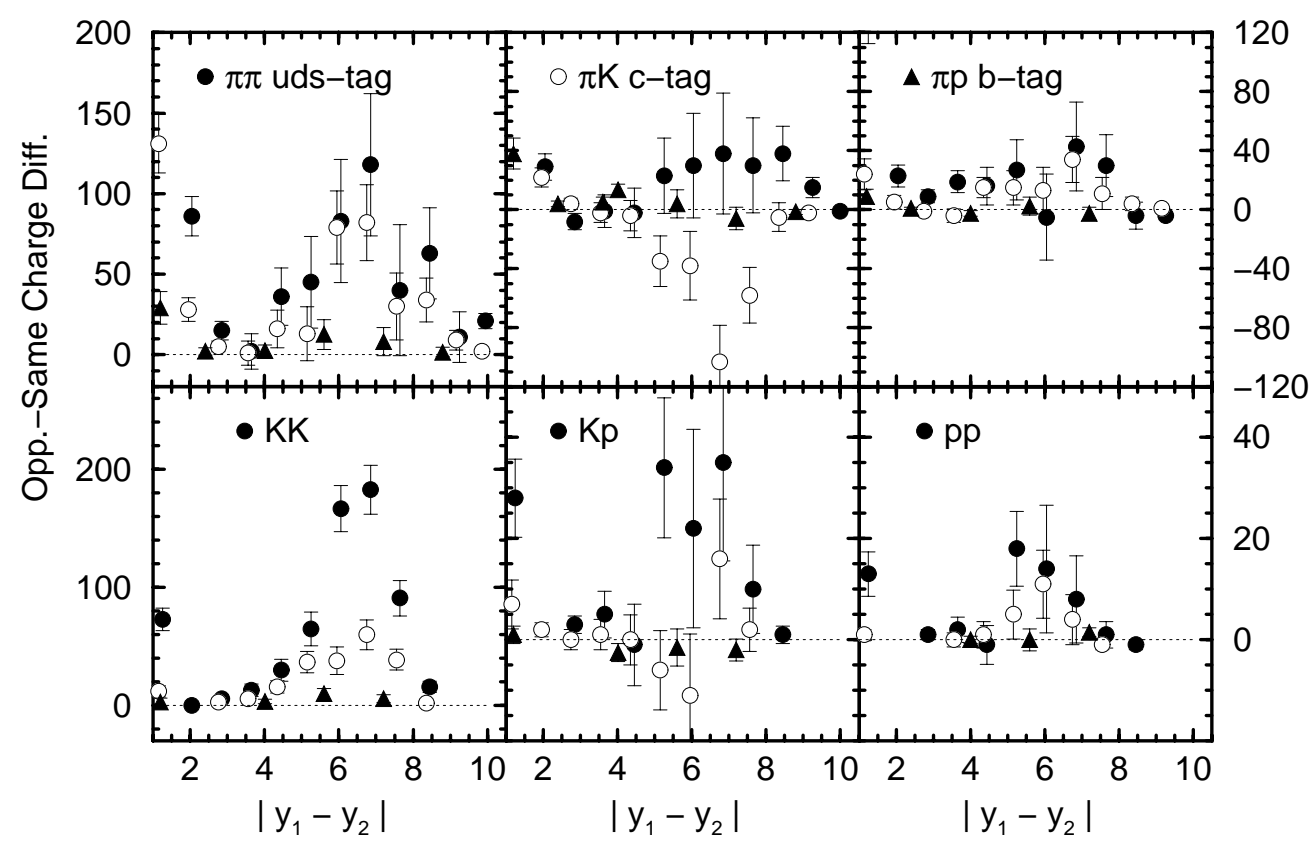

Figure 8: Distributions of $|\Delta y|$ for opposite-charge pairs minus those for same-charge pairs of $p>9 \mathrm{GeV} / c$ tracks, in the light-(dots), $c$-(circles), and $b$-tagged (triangles) samples.

the $q$ over the $\bar{q}$ direction. This excess is observed at all proton momenta so cannot be attributed simply to leading baryons. We have searched for similar signals for strangeness and charge ordering in the $K^{+} K^{-}$and $\pi^{+} \pi^{-}$samples, respectively, by isolating the light flavors and considering a variety of momentum bins. No significant effects are observed, possibly due to background from leading kaons and/or dilution due to resonance decays.

\section{Conclusions}

We use the excellent SLD vertexing and particle identificaton and the high SLC $e^{-}$beam polarization to make new QCD tests in the areas of event structure and jet fragmentation. In 3 -jet final states we tag jets as $b, \bar{b}$ or gluon jets and: measure the gluon energy spectrum over its full range, confirming a QCD prediction and limiting anomalous chromomagnetic couplings; find parity violation in $Z^{0} \rightarrow b \bar{b} g$ decays to be consistent with electroweak theory plus QCD; and perform new tests of T- and CP-conservation. Using 4-jet final states in which the two most collinear jets are tagged as $b / \bar{b}$, we measure the rate of gluon splitting into a $b \bar{b}$ pair in hadronic $Z^{0}$ decays, $g_{b \bar{b}}=0.00244 \pm 0.00059$ (stat.) \pm 0.00034 (syst.).

A technique for measuring $B$ hadron energies using only the charged tracks in a secondary vertex gives good efficiency and resolution at all energies. The full $B$-hadron energy distribution is measured precisely, allowing stringent tests of model predictions. We exclude a wide range of forms, constrain the shape tightly, and measure the average scaled energy precisely, $\left\langle x_{B}\right\rangle=0.710 \pm 0.003$ (stat.) \pm 0.005 (syst.) \pm 0.004 (shape) (Preliminary).

Using pairs of identified $\pi^{ \pm}, K^{ \pm}$and $\mathrm{p} / \overline{\mathrm{p}}$, we confirm local conservation of quantum numbers in jet fragmentation and observe local charge conservation between mesons and 

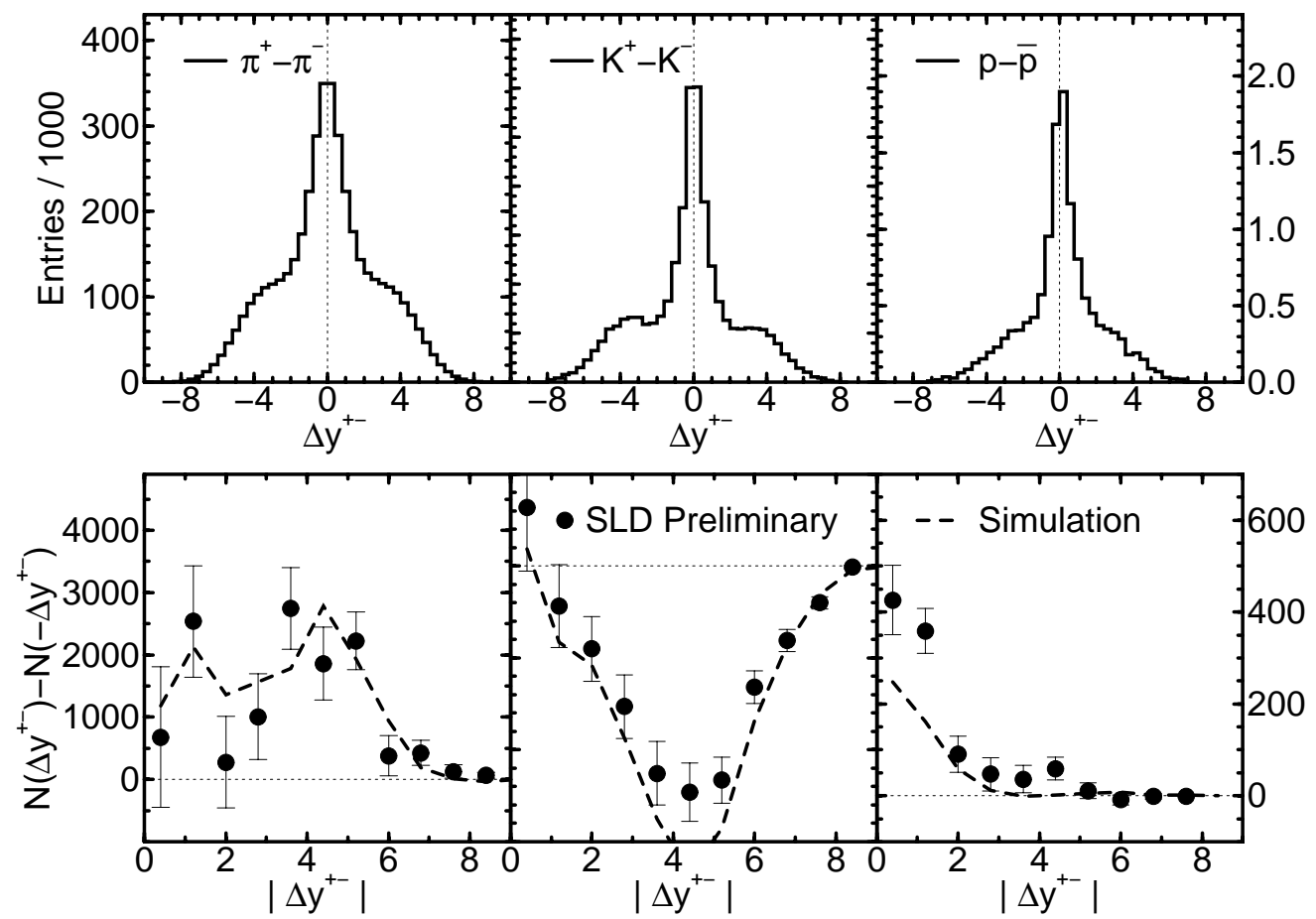

Figure 9: Distributions (top) of the ordered signed rapidity difference. Differences (bottom) between the positive and negative sides of each, along with JETSET predictions.

baryons, and between strange and nonstrange particles. Long range correlations observed for all pair types provide new information on leading particle production. The first study of ordered correlations in signed rapidity provides additional new insights, including the first direct observation of baryon number ordering along the quark-antiquark axis.

\section{References}

[1] SLD Collab., K. Abe et al., SLAC-Pub-8505.

[2] SLD Collab., K. Abe et al., Phys. Rev. Lett. 86 (2001) 962.

[3] SLD Collab., K. Abe et al., SLAC-Pub-8737, to appear in Physics Letters B.

[4] SLD Collab., K. Abe et al., SLAC-Pub-8504.

[5] SLD Collab., K. Abe et al., SLAC-Pub-8508.

[6] D.J. Jackson, Nucl. Inst. Meth. A388 (1997) 247.

[7] K. Abe, et al., Phys. Rev. Lett. 75 (1996) 4173.

[8] W. Bernreuther, A. Brandenburg, P. Uwer, Phys. Rev. Lett. 79 (1997) 189;

A. Brandenburg, P. Uwer, Nucl. Phys. B515 (1998) 279.

[9] T. Sjöstrand, Comp. Phys. Comm. 82 (1994) 74.

[10] T. Rizzo, Phys. Rev. D50 (1994) 4478.

[11] A. Brandenburg, L. Dixon and Y. Shadmi, Phys. Rev. D53 (1996) 1264. 


\section{${ }^{* *}$ List of Authors}

Koya Abe, ${ }^{(24)}$ Kenji Abe, ${ }^{(15)}$ T. Abe, ${ }^{(21)}$ I. Adam, ${ }^{(21)}$ H. Akimoto, ${ }^{(21)}$ D. Aston, ${ }^{(21)}$ K.G. Baird, ${ }^{(11)}$ C. Baltay, ${ }^{(30)}$ H.R. Band, ${ }^{(29)}$ T.L. Barklow, ${ }^{(21)}$ J.M. Bauer, ${ }^{(12)}$ G. Bellodi, ${ }^{(17)}$ R. Berger, ${ }^{(21)}$ G. Blaylock, ${ }^{(11)}$ J.R. Bogart, ${ }^{(21)}$ G.R. Bower, ${ }^{(21)}$ J.E. Brau, ${ }^{(16)}$ M. Breidenbach, ${ }^{(21)}$ W.M. Bugg, ${ }^{(23)}$ D. Burke, ${ }^{(21)}$ T.H. Burnett, ${ }^{(28)}$ P.N. Burrows, ${ }^{(17)}$ A. Calcaterra, ${ }^{(8)}$ R. Cassell, ${ }^{(21)}$ A. Chou, ${ }^{(21)}$ H.O. Cohn, ${ }^{(23)}$ J.A. Coller, ${ }^{(4)}$ M.R. Convery, ${ }^{(21)}$ V. Cook, ${ }^{(28)}$ R.F. Cowan, ${ }^{(13)}$ G. Crawford, ${ }^{(21)}$ C.J.S. Damerell, ${ }^{(19)}$ M. Daoudi, ${ }^{(21)}$ S. Dasu, ${ }^{(29)}$ N. de Groot, ${ }^{(2)}$ R. de Sangro, ${ }^{(8)}$ D.N. Dong, ${ }^{(13)}$ M. Doser, ${ }^{(21)}$ R. Dubois, ${ }^{(21)}$ I. Erofeeva, ${ }^{(14)}$ V. Eschenburg, ${ }^{(12)}$

E. Etzion, ${ }^{(29)}$ S. Fahey, ${ }^{(5)}$ D. Falciai, ${ }^{(8)}$ J.P. Fernandez ${ }^{(26)}$ K. Flood, ${ }^{(11)}$ R. Frey, ${ }^{(16)}$ E.L. Hart, ${ }^{(23)}$ K. Hasuko, ${ }^{(24)}$ S.S. Hertzbach, ${ }^{(11)}$ M.E. Huffer, ${ }^{(21)}$ X. Huynh, ${ }^{(21)}$ M. Iwasaki, (16) D.J. Jackson, ${ }^{(19)}$ P. Jacques, ${ }^{(20)}$ J.A. Jaros, ${ }^{(21)}$ Z.Y. Jiang, ${ }^{(21)}$ A.S. Johnson, ${ }^{(21)}$ J.R. Johnson, ${ }^{(29)}$ R. Kajikawa, ${ }^{(15)}$ M. Kalelkar, ${ }^{(20)}$ H.J. Kang, ${ }^{(20)}$ R.R. Kofler, ${ }^{(11)}$ R.S. Kroeger, ${ }^{(12)}$ M. Langston, ${ }^{(16)}$ D.W.G. Leith, ${ }^{(21)}$ V. Lia, ${ }^{(13)}$ C. Lin, ${ }^{(11)}$ G. Mancinelli, ${ }^{(20)}$ S. Manly, ${ }^{(30)}$ G. Mantovani, ${ }^{(18)}$ T.W. Markiewicz, ${ }^{(21)}$ T. Maruyama, ${ }^{(21)}$ A.K. McKemey, ${ }^{(3)}$ R. Messner, ${ }^{(21)}$ K.C. Moffeit, ${ }^{(21)}$ T.B. Moore, ${ }^{(30)}$ M. Morii, ${ }^{(21)}$ D. Muller, ${ }^{(21)}$ V. Murzin, ${ }^{(14)}$ S. Narita, ${ }^{(24)}$ U. Nauenberg, ${ }^{(5)}$ H. Neal, ${ }^{(30)}$ G. Nesom, ${ }^{(17)}$

N. Oishi, ${ }^{(15)}$ D. Onoprienko, ${ }^{(23)}$ L.S. Osborne, ${ }^{(13)}$ R.S. Panvini, ${ }^{(27)}$ C.H. Park, ${ }^{(22)}$ I. Peruzzi, ${ }^{(8)}$ M. Piccolo, ${ }^{(8)}$ L. Piemontese, ${ }^{(7)}$ R.J. Plano, ${ }^{(20)}$ R. Prepost, ${ }^{(29)}$ C.Y. Prescott, ${ }^{(21)}$ B.N. Ratcliff, ${ }^{(21)}$ J. Reidy, ${ }^{(12)}$ P.L. Reinertsen, ${ }^{(26)}$ L.S. Rochester, ${ }^{(21)}$ P.C. Rowson, ${ }^{(21)}$ J.J. Russell, ${ }^{(21)}$ O.H. Saxton, ${ }^{(21)}$ T. Schalk, ${ }^{(26)}$ B.A. Schumm, ${ }^{(26)}$ J. Schwiening, ${ }^{(21)}$ V.V. Serbo, ${ }^{(21)}$ G. Shapiro, ${ }^{(10)}$ N.B. Sinev, ${ }^{(16)}$ J.A. Snyder, ${ }^{(30)}$ H. Staengle, ${ }^{(6)}$ A. Stahl, ${ }^{(21)}$ P. Stamer, ${ }^{(20)}$ H. Steiner, ${ }^{(10)}$ D. Su, ${ }^{(21)}$ F. Suekane, ${ }^{(24)}$ A. Sugiyama, ${ }^{(15)}$ S. Suzuki, ${ }^{(15)}$ M. Swartz, ${ }^{(9)}$ F.E. Taylor, ${ }^{(13)}$ J. Thom, ${ }^{(21)}$ E. Torrence, ${ }^{(13)}$ T. Usher, ${ }^{(21)}$ J. Va'vra, ${ }^{(21)}$ R. Verdier, ${ }^{(13)}$ D.L. Wagner, ${ }^{(5)}$ A.P. Waite, ${ }^{(21)}$ S. Walston, ${ }^{(16)}$ A.W. Weidemann, ${ }^{(23)}$ E.R. Weiss, ${ }^{(28)}$ J.S. Whitaker, ${ }^{(4)}$ S.H. Williams, ${ }^{(21)}$ S. Willocq, ${ }^{(11)}$ R.J. Wilson, ${ }^{(6)}$ W.J. Wisniewski, ${ }^{(21)}$ J.L. Wittlin, ${ }^{(11)}$ M. Woods, ${ }^{(21)}$ T.R. Wright, ${ }^{(29)}$ R.K. Yamamoto, ${ }^{(13)}$ J. Yashima, ${ }^{(24)}$ S.J. Yellin, ${ }^{(25)}$ C.C. Young, ${ }^{(21)}$ H. Yuta. ${ }^{(1)}$

(1) Aomori University, Aomori , 030 Japan, (2) University of Bristol, Bristol, U.K.,

(3) Brunel University, Uxbridge, Middlesex, UB8 3PH United Kingdom,

(4) Boston University, Boston, Massachusetts 02215,

(5) University of Colorado, Boulder, Colorado 80309,

(6) Colorado State University, Ft. Collins, Colorado 80523,

(7) INFN Sezione di Ferrara and Universita di Ferrara, I-44100 Ferrara, Italy,

(8) INFN Laboratori Nazionali di Frascati, I-00044 Frascati, Italy,

(9) Johns Hopkins University, Baltimore, Maryland 21218-2686,

(10) Lawrence Berkeley Laboratory, University of California, Berkeley, California 94720,

(11) University of Massachusetts, Amherst, Massachusetts 01003,

(12) University of Mississippi, University, Mississippi 38677,

(13) Massachusetts Institute of Technology, Cambridge, Massachusetts 02139,

${ }^{(14)}$ Institute of Nuclear Physics, Moscow State University, 119899, Moscow Russia,

${ }^{(15)}$ Nagoya University, Chikusa-ku, Nagoya, 464 Japan,

(16) University of Oregon, Eugene, Oregon 97403, 
(17) Oxford University, Oxford, OX1 3RH, United Kingdom,

(18) INFN Sezione di Perugia and Universita di Perugia, I-06100 Perugia, Italy,

${ }^{(19)}$ Rutherford Appleton Laboratory, Chilton, Didcot, Oxon OX11 OQX United Kingdom,

${ }^{(20)}$ Rutgers University, Piscataway, New Jersey 08855,

(21) Stanford Linear Accelerator Center, Stanford University, Stanford, California 94309,

${ }^{(22)}$ Soongsil University, Seoul, Korea 156-743,

(23) University of Tennessee, Knoxville, Tennessee 37996,

(24) Tohoku University, Sendai 980, Japan,

(25) University of California at Santa Barbara, Santa Barbara, California 93106,

(26) University of California at Santa Cruz, Santa Cruz, California 95064,

(27) Vanderbilt University, Nashville, Tennessee 37235,

(28) University of Washington, Seattle, Washington 98105,

(29) University of Wisconsin, Madison, Wisconsin 53706,

(30) Yale University, New Haven, Connecticut 06511. 